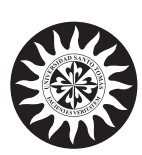

\title{
Estudios sobre medio ambiente y sostenibilidad: una mirada desde Colombia
}

Hugo Fernando Guerrero Sierra

María Eugenia Vega

Pedro Mauricio Acosta Castellanos 


\section{Estudios sobre medio ambiente y sostenibilidad: una mirada desde Colombia}

Hugo Fernando Guerrero Sierra / María Eugenia Vega / Pedro Mauricio Acosta Castellanos.

Universidad Santo Tomás..

Tunja: 2018

ISBN: 978-958-5471-12-2

1. Sostenibilidad, Medio Ambiente, Colombia, América Latina, Estudios Interdisciplinares

Estudios sobre medio ambiente y sostenibilidad: una mirada desde Colombia Primera edición, noviembre de 2018

ISBN: 978-958-5471-12-2

Hugo Fernando Guerrero Sierra, María Eugenia Vega, Pedro Mauricio Acosta Castellanos.

Corrección de Estilo:

Fray Ángel María Beltrán N., O.P.

Todos los derechos reservados conforme a la ley.

Se permite la reproducción citando fuente.

El pensamiento que se expresa en esta obra, es exclusiva responsabilidad de los autores y no compromete la ideología de la Universidad Santo Tomás.

\section{Diagramación e impresión:}

Búhos editores Ltda.

Calle $57 \mathrm{~N}^{\circ} 9-36$

Tunja - Boyacá

buhosedi@gmail.com

\section{Ediciones Usta}

Universidad Santo Tomás

2018

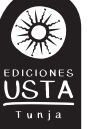

Departamento

Ediciones Usta Tunja

Queda prohibida la reproducción parcial o total de este libro por cualquier proceso reprográfico o fónico, especialmente por fotocopia, microfilme, offset o mimeógrafo. Ley 23 de 1982. 


\section{BIOGRAFÍA EDITORES ACADÉMICOS}

\section{Hugo Fernando Guerrero Sierra Ph.D:}

Doctor Cum Laude en Relaciones Internacionales y Globalización por la Universidad Complutense de Madrid. Abogado de la Universidad Nacional de Colombia. Ha adelantado su actividad investigadora y docente en diferentes centros universitarios de España, Estados Unidos y Colombia. Autor y editor de diferentes libros y artículos en las áreas del análisis del conflicto, gobernanza y las relaciones internacionales. Actualmente es Docente-Investigador y Director del Grupo Interdisciplinar de Investigación en Política y Relaciones Internacionales de la Universidad de La Salle.

\section{María Eugenia Vega Mg:}

Politóloga Universidad de Buenos Aires. MSc en Defensa Nacional, Escuela de Defensa de la República Argentina. Doctoranda en Ciencias Sociales Universidad de Buenos Aires. Docente de la Universidad de Buenos Aires, profesora visitante e investigadora en la Maestría en Ciencia Política de la Universidad de Los Andes, Mérida, Venezuela. Actualmente profesora investigadora Profesora investigadora de tiempo completo del Programa de Negocios Internacionales. Universitaria Agustiniana (UniAgustiniana). Bogotá DC.

\section{Pedro Mauricio Acosta Castellanos Mg:}

Decano de la Facultad de Ingeniería Ambiental de la Universidad Santo Tomás Seccional Tunja. Ingeniero Civil, Especialista en Comportamiento y Conservación del Recurso Hídrico, Magister en Ingeniería Civil con Énfasis en Hidroambiental de la niversidad Santo Tomás, Especialista en Ingeniería Sanitaria y Ambiental y Magister en Ingeniería Sanitaria de la Universidad de Buenos Aires. Docente e investigador a nivel universitario, director del grupo de investigación en ciencias ambientales y naturales de la Universidad Santo Tomás Seccional Tunja. 



\section{Contenido}

Presentación

Introducción 11

\section{PRIMERA PARTE DEL CONFLICTO ARMADO A LA PAZ AMBIENTAL}

CAPÍTULO I. Paz ambiental y paz territorial: Ios desafíos de Colombia para el postconflicto

CAPÍTULO II. De la evaluación económica a la evaluación sociopolítica de las políticas públicas ambientales y agrarias en Colombia.....

CAPÍTULO III. Discordancia jurídica sobre un tema ambiental en Colombia.

La naturaleza como sujeto de derechos y las tasas retributivas

CAPÍTULO IV. Los conflictos ambientales: una desapercibida fuente de desplazamiento en Colombia

\section{SEGUNDA PARTE \\ EXPERIENCIAS LOCALES EN MEDIO AMBIENTE Y SOSTENIBILIDAD.}

CAPÍTULO V. El borde urbano: límite virtual sobre la fragilidad rural.....

123

CAPÍTULO VI. Alternativas para el desarrollo sostenible de la zona de reserva campesina. Municipio de Cabrera - Cundinamarca.

CAPÍTULO VII. El impacto del sector minero energético en el desarrollo social de las regiones: en caso del Catatumbo, Colombia.....

CAPÍTULO VIII. La gestión pública del servicio de agua desde un enfoque de desarrollo sostenible: el caso de Florencia - Caquetá....

CAPÍTULO IX. Conflictos socioambientales y deterioro de la salud colectiva en la cuenca del río Tunjuelo 


\section{TERCERA PARTE PEDAGOGÍA AMBIENTAL Y PRÁCTICAS SOSTENIBLES}

CAPÍTULO X. Información y educación para la sostenibilidad de espacios ambientales protegidos en Colombia.....

CAPÍTULO XI. Condiciones para la difusión de los sistemas fotovoltaicos residenciales en Colombia 301

CAPÍTULO XII. Consumo responsable de servicios públicos domiciliarios en Colombia 323

CAPÍTULO XIII. Generación de residuos sólidos urbanos: determinantes para el caso colombiano

CAPÍTULO XIV. Sostenibilidad, competitividad y Logística Inversa. Prácticas de la Industria Editorial y Comunicación Gráfica colombiana

\section{CUARTA PARTE PENSAR EL MEDIO AMBIENTE: UN DESAFÍO EN AMÉRICA LATINA}

CAPÍTULO XV. Una mirada ambiental a la Alianza del Pacifico 389

CAPÍTULO XVI. Desafíos para la construcción de una agenda ambiental en el regionalismo. El caso de la Alianza del Pacífico

CAPÍTULO XVII. Cuando la tierra explotó: comunidad y territorio cambiantes por la extracción de petróleo en México

Epílogo. Un texto necesario... pero insuficiente 483 


\section{Presentación}

E

n los últimos lustros, el tema ambiental ha adquirido una mayor significación para la humanidad y está relacionado de manera interdisciplinar con diversos campos del saber. La toma de decisiones en el estamento público de quienes determinan acciones en el ámbito científico, jurídico, político y económico son importantes, pues cada acción que aborde lo relacionado al medio ambiente y la sostenibilidad concierne también a la vida humana, a su preservación y continuidad.

En los orígenes de la tradición dominicana, San Alberto Magno abrió una línea importante de reflexión académica para la humanidad al profundizar sobre los conocimientos de la naturaleza e indagar sus principios universales. La exigencia académica del maestro de Tomás de Aquino, condensa así una articulación de las ciencias naturales con el modo universal del conocimiento de las fuentes en la búsqueda de la verdad. La naturaleza se reconoce como lugar privilegiado de conocimiento y de experimentación que por la vía de la razón humana se persigue inteligir lo real, dando así cabida a iniciativas científicas, valoradas a lo largo de la historia.

La Carta Encíclica LAUDATO SI, del Papa Francisco, Sobre el Cuidado de la Casa Común, pone de relieve a toda la humanidad un mensaje de interiorización sobre nuestra manera de relacionarnos con la naturaleza. La reflexión deja ver abiertamente la preocupación en que vivimos actualmente sobre el cuidado del medio ambiente; tensión que se agudiza día a día por el consumo desmedido de los recursos naturales, poniendo en riesgo el equilibrio de la naturaleza. El inicio de la Encíclica marca un llamado de lenguaje, de espiritualidad y de nueva relación con la naturaleza, calcada del Cántico de las Creaturas de San Francisco: "Alabado seas, mi Señor, por la hermana nuestra madre tierra".

Hoy, más que nunca, se conmina a la academia a tomar parte importante en los temas ambientales, conduciendo a una reflexión seria, ética y crítica que oriente las acciones concretas y prácticas de nuestro actuar a partir del binomio sociedad - naturaleza. En este sentido, la Universidad Santo Tomás refuerza este compromiso para 
que, desde los distintos programas académicos que ofrece la institución, en vista a la vida profesional y la realización personal de los estudiantes, pueda labrar en estos espacios académicos una vocación ambiental. Es de reconocer que en el momento presente hay una alta producción académica en torno al desarrollo sostenible y medio ambiente, lo que explica la importancia de la temática. Además, la presencia de programas académicos de distintas universidades en el país, con un marcado interés por lo ambiental, sirve de base para fraguar unos ejes claves de reflexión en la profundización científica y profesional concerniente a la acción humana y su impacto en la naturaleza.

A nivel regional, destacamos la presencia de la Facultad de Ingeniería Ambiental de la Universidad Santo Tomás - Seccional Tunja - para contribuir in situs con la formación profesional en esta área del conocimiento. Desde hace tres años, la puesta en acción de una comunidad académica, nos permite animar una reflexión de conjunto más acorde para dar respuesta a nuestras particularidades. Ahora se ve importante y conveniente fortalecer una colección en donde se pueda consolar la producción escrita de investigadores, docentes, estudiantes que tienen que ver con la vida curricular de una Facultad desde una perspectiva interdisciplinar y en diálogo constante con pares de diferentes orígenes académicos, profesionales, geográficos y sociales. Esto le permite al lector una mirada amplia sobre el tema.

En este sentido, se presenta a la comunidad académica la Colección Temas Ambientales que plasma el esfuerzo de un grupo de investigadores para establecer algunas ideas claves que fecundan la enseñanza de la transversalidad inherente a los estudios ambientales. Esta Colección permite establecer los inicios de una reflexión que se irá consolidando a lo largo de los años, y permitirá a la vez tener una mirada amplia de los distintos fenómenos que enmarca cada problemática para nutrir el currículo de la Facultad y, en general, de la proyección de la Universidad como interlocutor válido respecto de los debates medioambientales. Este ejercicio de publicación genera además un acercamiento a la responsabilidad que se tiene con el cuidado y sustentabilidad del uso de los recursos naturales, despertando la conciencia para un mayor conocimiento de discernimiento de nuestro rol y acción frente a la naturaleza. La nueva generación, impregnada de globalización y tecnología, tendrá la misión de repen- 
sar la presencia humana y su intervención en la casa común que todos habitamos.

El presente libro de la Colección naciente es una compilación de investigación referidas a nuestra situación de Colombia en el contexto latinoamericano, marcada hoy por la experiencia de la paz, luego de un conflicto de varias décadas de nuestra historia. Al igual, se recoge allí las experiencias locales del medio ambiente y la sostenibilidad, mediadas por la pedagogía y las orientaciones del manejo de los recursos naturales.

Resalto la iniciativa de los coordinadores, Dr. Hugo Fernando Guerrero Sierra, Dra. María Eugenia Vega e Ing. Pedro Mauricio Acosta Castellanos, por condensar la temática para su publicación y por su celo académico para que las reflexiones de tan importante problemática sean divulgadas en medio Universitario. Agradezco la motivación y el marcado interés por la creación de la Colección Temas Ambientales del Decano fundador de la Facultad de Ingeniería Ambiental en nuestro claustro universitario de Tunja.

Fr. Samuel Elías FORERO BUITRAGO, O.P. Decano de División de Ingenierías y Arquitectura Universidad Santo Tomás - Seccional Tunja 21 de mayo 2018 



\section{Introducción}

L

a atención puesta por la comunidad internacional sobre los temas ambientales no constituye ninguna novedad; desde los años 70, han proliferado un sinnúmero de tratados, protocolos, acuerdos, cumbres, organismos e instituciones en la búsqueda de lograr acciones concertadas entre los Estados a fin de enfrentar una problemática global. Sin embargo, y ya bien entrado el siglo XXI, su falta de operatividad muestra la distancia que existe entre los objetivos propuestos por las distintas instancias y los instrumentos que posibilitarían al menos paliar parte del problema. Si bien son innegables los avances -muchos y variados, sobre todo en ciertas regiones del planeta-, los temas ambientales parecen haber caído en el campo de la retórica.

El mundo asiste, como un espectador impávido, a las catástrofes naturales y humanas producto de la degradación ambiental, la escasez de agua, alimentos, tierras, la desaparición de especies de toda índole, la contaminación y el cambio climático. Un fenómeno multidimensional, transversal y global que se constituye en el factor por excelencia que demuestra la incongruencia entre las fronteras legales internacionales y las redes ecológicas. Mucho menos puede dudarse del creciente vínculo entre el sector económico y el medioambiental en la medida en que las condiciones de la producción en masa amenazan la estructura del ecosistema planetario. Aunque estos elementos afectan a la humanidad en su conjunto, son justamente los habitantes de los países en desarrollo los que sienten sus efectos por cuanto dependen directamente de actividades primarias.

Además, el modelo de desarrollo de las últimas décadas que, junto a los impactos ambientales de la liberalización del comercio, han reprimarizado a las economías más vulnerables, como es el caso de América Latina. Sobre las bases de su propia tradición extractivista, la región ha generado incrementos en los flujos internacionales de sus economías, pero con el costo de dejar a su paso una brecha ambiental cada vez más extensa. Factores que no pueden dejarse de lado, como la presencia del crimen organizado que a través de mercados negros, minería ilegal, deforestación agresiva, tráfico de armas, especies y personas que a su vez se catalizan con el conflic- 
to armado colombiano, que encuentran el caldo de cultivo ideal en situaciones de inequidad social, corrupción y debilidad institucional, necesariamente deben ser pensados en perspectiva ambiental con visión latinoamericana.

Así las cosas, ha sido sobre todo la academia la que ha encendido la luz de alarma sobre estos temas. Es en este marco, donde la presente propuesta propende a la discusión de estas preocupaciones, ya que pareciese que, salvo honrosas excepciones, estas no son parte de los temas urgentes a tratar por las agendas políticas. Los abordajes que aquí presentan los autores abarcan diversos aspectos que dan cuenta, no sólo de la referida multidimensionalidad de la problemática, sino que también ilustran la necesidad de acciones conjuntas en todos los niveles, desde lo global a lo regional, desde lo subregional a lo nacional, desde el poder central del Estado a las acciones políticas encabezadas por la sociedad civil.

Como la idea central y guía de esta propuesta es propender a la discusión inter y transdisciplinar, desde su propia estructura ésta queda representada; así, el libro se organiza en cuatro grandes secciones temáticas que a su vez dialogan desde los distintos saberes, narrativas y metodologías.

La primera parte, del conflicto armado a la paz ambiental, consta de cuatro capítulos. En el primero, David González Cuenca, Douglas Molina y Ana María Montes centran su análisis en exponer los desafíos que representa para Colombia el posconflicto frente a un modelo de paz territorial y paz ambiental. Con un abordaje cualitativo, los autores desarrollan y profundizan estos conceptos al aplicarlos al contexto colombiano. Esto con el fin de destacar los retos para la implementación de programas de paz territorial, en los que se espera que sean los propios actores de la sociedad civil los protagonistas centrales y que el postconflicto no afecte los ecosistemas que han permanecido intactos.

En el capítulo II, Adriana Otálora y Omar Vivas Cortés afirman que el tránsito de una evaluación con énfasis económico a una evaluación que considere a los actores políticos que participan en la implementación de políticas ambientales, agrarias y de otros asuntos en Colombia, a partir de un enfoque sociopolítico, permite visibilizar 
la presencia y acción política de grupos o colectivos, más o menos institucionalizados, cuya eficacia marca los atributos políticos de los productos de la acción pública estructurada que toma como referente la política. Este aporte presenta los resultados del análisis que hacen los investigadores sobre las evaluaciones realizadas por parte del Departamento Nacional de Planeación a algunas políticas nacionales, para ello, problematiza el esquema de evaluación utilizado al concebir que la mirada de proceso concentrada en cifras inhibe la consideración del juego político y la presencia de los actores, juego que se convierte en una caja negra; tras la revisión de las evaluaciones se evidencia que los atributos políticos no son valorados, hecho que dista de los planteamientos propios de la nueva gobernanza, la coproducción de bienes y servicios públicos, la democracia en profundidad que concibe a un ciudadano activo e integrado, e incluso, que la gobernabilidad, como capacidad de logro, enfrente una franja opaca.

Por su parte, John Rúa Castaño en el capítulo III, se interroga desde un punto jurídico acerca de la completitud y coherencia del sistema jurídico colombiano en lo que refiere a las normas legales que regulan los vertidos y las emisiones industriales. Basa su análisis en el caso del río Atrato, el que fue reconocido como entidad sujeta de derechos a la protección, conservación, mantenimiento y restauración. Discute y recomienda sustituir las tasas retributivas y compensatorias, ya que éstas se han vuelto fuente rentística de las Corporaciones Autónomas Regionales y a fin de evitar los conflictos de intereses. Finalmente, reflexiona acerca del Estado Social de Derecho y un Derecho Ambiental colombiano que prioriza "quien contamina, paga" antes de reconocer a la naturaleza como un sujeto de derechos.

Mientras que Edward Garzón y Kevin Suárez plantean, en el capítulo IV, los problemas relativos a la ausencia de definiciones acerca de los desplazamientos forzados por causas ambientales, que si bien pueden traer consigo una serie de consecuencias, el desplazamiento forzado pasa desapercibido por la inexistencia de nuevas categorías o la ambigüedad de la categoría existente. Sin una categoría acorde al problema, existen limitaciones para la protección de la población afectada por parte de los Estados y/o las organizaciones internacionales. Por tanto, el propósito de esta investigación es analizar críticamente la discusión en torno a la inexistencia de una definición acerca 
del desplazado ambiental en relación a los conflictos ambientales en Colombia (2016).

Experiencias locales en medio ambiente y sostenibilidad, constituye la segunda parte de este trabajo. Se encuentran aquí múltiples miradas y experiencias que toman distintas problemáticas ambientales, en su mayoría desde contextos micro. La excepción se da en el primer trabajo de esta sección, donde Laura Zimmerman y Tomás Neu, desde los estudios urbanos, critican cómo la evolución disciplinar y los vacíos institucionales conllevaron resultados de índole económica perdiéndose consideraciones de forma y estructura, con relación al hábitat y al medio ambiente. Así, la posibilidad de confirmar procesos de pertenencia y permanencia de asentamientos persiguen lógicas específicas donde morfología urbana y tipología arquitectónica determinan calidad de vida. Con un texto que ilustra funcionalidades sobre el manejo de bordes urbanos y paisajes intermedios, así como la manera de integrarlos coherentemente al crecimiento urbano y preservación ambiental, los autores llevan por delante la premisa de que proposiciones e intervenciones urbanas sistémicas deben encauzar y formalizar gobernabilidad, gobernanza y política pública como beneficio generalizado.

En el capítulo IV, José Armando Hernández, Laura Castiblanco y Alejandra Castillo proponen el análisis de las formas de financiación estatal ofrecidas al sector agricultor a nivel nacional, y a partir de esto, evaluar el mecanismo crediticito idóneo para otorgar a los campesinos ubicados en zona de reserva campesina de Cabrera-Cundinamarca, teniendo en cuenta las características demográficas, económicas y crediticias de dicha población. Para esto, diseñan una propuesta de financiación, en donde componentes de conservación, manejo, control y aprovechamiento de los recursos naturales, infraestructura, producción limpia, educación ambiental, comercialización y servicios, permitan el desarrollo ambiental, social, productivo y territorial de la región de Cabrera.

Continúan la secuencia Amanda Vargas y Javier García, quienes analizan en qué medida el mayor dinamismo del sector minero-energético, se traduce en mejores indicadores de desarrollo social de los ciudadanos del Norte de Santander, más específicamente del Catatumbo. Enfatizan que el modelo extractivista en Colombia debe ser examinado con detenimiento para evaluar los impactos ambien- 
tales y sociales que genera, ya que la estrategia de desarrollo deberá ampliarse para incluir otras dimensiones del crecimiento amén del PIB. Concluyen que la región del Catatumbo presenta los graves problemas de otras zonas rurales en el país, verdaderos desafíos para el desarrollo económico y social, los que deben ser abordados en conjunto para el diseño de políticas públicas en la implementación del Acuerdo Final del Conflicto Armado en Colombia.

En la gestión pública del servicio del agua en el marco del desarrollo sostenible en Florencia, Caquetá: Paola Villanueva, Hugo Guerrero y Pedro Mauricio Acosta analizan el estado actual de la gestión pública del agua y su relación con la participación comunitaria en un marco de desarrollo sostenible en este. Con esta finalidad, realizan una revisión teórica sobre las categorías de: agua como bien público, desarrollo sostenible y gestión pública enfocada en la Nueva Gestión Pública. Para el análisis de caso, se seleccionaron las entidades con mayor injerencia en el tema y se aplicó el instrumento de la entrevista estructurada a los funcionarios asignados. Asimismo, se hizo una caracterización organizacional, de articulación y de funcionamiento de las entidades. Finalmente, se recolectó y analizó información sobre los mecanismos de participación y la percepción social sobre la gestión pública del agua, mediante encuesta aplicada a una muestra del 30\% del total de Presidentes de Juntas Comunales del municipio. Fruto de este ejercicio, los autores lograron determinar que en Florencia la administración municipal, dentro de un modelo de gestión pública tradicional, viene prestando el servicio público del agua potable de manera externalizada, a través de una empresa de economía mixta, autónoma y regida por el derecho privado; con ausencia de un control real, donde la participación ciudadana y el desarrollo sostenible son sólo formalismos que no tienen una aplicación con alcance material.

Cierra esta sección Giovanni Mora, quien en el capítulo IX muestra un caso de la relación entre los conflictos socio-ambientales y el deterioro de la salud colectiva de los pobladores a través del estudio de la cuenca alta y media del río Tunjuelo, Bogotá. Con estos fines, el autor sitúa históricamente la construcción del conflicto ambiental y sanitario para luego pasar a analizar el proceso de urbanización y los efectos de depredación en la cuenca media y visualizar las causas y consecuencias ambientales y sanitarias del relleno sanitario ubicado en cercanías de las localidades de Usme y Ciudad Bolívar. Su objetivo central, es actualizar el mapa de los conflictos socio-ambientales y 
sanitarios en Bogotá, alimentar la comprensión socio-histórica de los mismos teniendo como telón de fondo las relaciones sociales de tipo capitalista que se tejen en la dialéctica sociedad-naturaleza.

Pedagogía ambiental y prácticas sostenibles se titula la tercera parte de libro, inaugurada por Johann Pirela y Yamely Almarza, quienes consideran que los discursos y prácticas sobre la sostenibilidad de espacios ambientales protegidos en Colombia deben estructurarse a partir de racionalidades que integren acciones informativas y educativas, orientadas hacia el desarrollo de una consciencia ética y crítica en torno a la posibilidad de utilización y apropiación del medioambiente como territorio en que convergen además de elementos ecológicos, componentes que configuran la espacialidad, entendida como totalidad de entramado de relaciones complejas con una alta potencialidad informativa y educativa, con miras, además, a desarrollar competencias ciudadanas, vinculadas con la protección y defensa del medio ambiente. A partir de estas premisas, los autores ponen de manifiesto en el capítulo X la contribución que puede hacer la información y la educación como procesos esenciales a partir de los cuales se realicen aportes a los debates contemporáneos sobre el medioambiente y la sostenibilidad, generando productos y servicios que maximicen el uso de los recursos ambientales y su aprovechamiento en procesos formativos. La metodología consistió en un enfoque cualitativo, de tipo descriptivo, con técnicas de investigación documental, como el análisis de contenido, la comparación de las dos perspectivas asumidas como ejes transversales: información y educación; en función de las cuales se formulan propuestas de uso responsable y sostenible de espacios ambientales protegidos.

I capítulo XI: Condiciones para la difusión de los sistemas fotovoltaicos residenciales en Colombia, en el que Carlos Morales estudia el caso de los sistemas de generación solar fotovoltaica usando un incentivo conocido como tarifas de alimentación, el que ha resultado muy exitoso en diversas partes del mundo, por cuanto permite que cualquier unidad económica, incluidas las viviendas familiares, generen energía proveniente de fuentes renovables y así paguen un precio preferencial que incentive una mayor generación. Afirma el autor que, en el caso de Colombia, estas posibilidades prácticamente no han entrado en el debate público. Para esto, toma en consideración los casos de Bogotá y San Andrés y Providencia; mientras en el primero no asoman datos costo eficientes, en el segundo resulta promete- 
dor. Concluye que el proceso de adopción de estos sistemas debe hacerse con la participación de las comunidades del departamento para que ellas mismas contribuyan en el diseño de la estructura de generación, ya que la adopción de esquemas de energía fotovoltaica con generación distribuida representa una plataforma para estimular la innovación con impacto local.

Por otra parte, Ramiro Rodríguez y Kelly Johana Díaz, en el capítulo XII, responden a la pregunta ¿Cuál es el comportamiento en el consumo y uso responsable y sostenible de servicios públicos en Colombia? El documento revisa el consumo responsable en el marco de los Objetivos de Desarrollo Sostenible propuestos en el 2015, particularmente en el tema de los servicios públicos. Para esto, se caracteriza el consumo de servicios públicos identificando prácticas sostenibles, discriminado por regiones y departamentos, de tal manera que es posible identificar las poblaciones con consumos responsables y no responsables de servicios públicos. Adicionalmente y con una metodología cuantitativa se cuantifica el consumo sostenible de servicios públicos, para lo que se utiliza la base de datos microanonimizada recolectada por el DANE a nivel nacional denominada "Encuesta de Calidad de Vida ECV 2016", la cual dispone de un módulo para los servicios públicos domiciliarios.

También, desde una visión propia de las Ciencias Económicas, en el capítulo XIII, María del Pilar Sánchez y María Gabriela Ramos, consideran que el manejo adecuado de residuos sólidos puede constituirse en un factor determinante para mejorar las condiciones medio ambientales de las ciudades, con repercusiones favorables tanto para el ambiente como para todos los agentes económicos involucrados en esta actividad. Se analizan los determinantes de la generación de residuos sólidos urbanos en los departamentos de Colombia, a través de un modelo de datos panel, con el fin de establecer las variables que son estadísticamente significativas para determinar su generación, y de esta forma, proponer alternativas que aporten al diseño de políticas públicas.

Finalizando el apartado, Andrea Cely y Julio César Ducón, identifican algunas formas en que son aprovechados residuos generados en la Industria Editorial y Comunicación Gráfica en el contexto colombiano y las posibles ventajas competitivas que pueden derivarse de la implementación de procesos de logística inversa en el marco 
de la sostenibilidad ambiental. El método utilizado para la obtención de resultados fue exploratorio-descriptivo, en el que se aplicaron técnicas de observación no participante para la consecución de información primaria, mientras que la información proveniente de fuentes secundarias, se obtuvo principalmente de consultas en documentación institucional e informes de agremiaciones del sector por medio de las cuales se hizo una descripción de los diferentes modelos implementados. Esto les ha permitido concluir que, a pesar de ser una industria caracterizada por requerir insumos y materias primas de difícil aprovechamiento residual, ha ido avanzando en la toma de conciencia sobre los impactos ambientales generados, a tal punto que algunas de las principales empresas han adoptado diferentes modelos basados en la perspectiva de la logística inversa obteniendo importantes beneficios.

La cuarta y última línea, denominada, Pensar el medio ambiente: un desafío en América Latina, brinda una perspectiva más amplia de Colombia en relación con su región. El capítulo XV de la autoría de Sara Quintero y Alexander Fula comienza con una revisión a la agenda doméstica de los gobiernos de la Alianza del Pacifico (México, Colombia, Perú y Chile), para determinar la transversalidad de las políticas domésticas con los compromisos de la cooperación en el eje de la Alianza del Pacifico. Este insumo, posteriormente permitirá determinar si la cooperación en materia ambiental obedece al principio de la conservación de recursos ambientales, para el comercio internacional (ámbito racional-económico, costo beneficio) o si por el contario corresponde al compromiso internacional ligado a los regímenes internacionales sobre el cambio climático (idealista-Kantiano, basado en los regímenes del Derecho Internacional).

En el capítulo XVI, María Eugenia Vega y Hadrien Lafosse, consideran las relaciones existentes entre el regionalismo latinoamericano y el medio ambiente, tomando como caso de estudio a la Alianza del Pacífico desde un abordaje que utiliza la técnica del análisis de contenido. En principio, se critica la ausencia de estudios suficientes y actualizados que aborden la interrelación entre estos aspectos, así como de lo exiguo de lo conseguido hasta la actualidad en materia ambiental por parte de la Alianza del Pacífico. Así, y a fin de proponer la necesidad de una agenda ambiental común para este esquema de integración, los autores analizan las agendas nacionales ambientales de los cuatro miembros. Al encontrar convergencias y/concordancias 
tan amplias entre las mismas, proponen que éstas podrían convertirse en la plataforma para que el denominado regionalismo neoliberal dé un giro hacia el tratamiento de las problemáticas medioambientales y no sólo a los netamente comerciales, como tradicionalmente lo ha hecho.

Por último, Svenja Schöneich, muestra cómo por más de 50 años la extracción de hidrocarburos ha sido una parte importante de la vida cotidiana en la comunidad campesina Emiliano Zapata en Veracruz México. Entiende la autora que la presencia de la empresa, así como las actividades de extracción en general, están profundamente inscritas en el paisaje y en las condiciones de vida de sus habitantes ya que la contaminación, los daños en los terrenos y los ríos, la construcción de la infraestructura, las nuevas oportunidades de educación y de trabajo, han cambiado a la comunidad y su relación con su entorno natural de una manera significativa. Sin embargo, los nuevos desafíos provocados por la Reforma Energética Mexicana 2013-2014, que abre el sector de hidrocarburos para la inversión privada y termina con el monopolio de PEMEX, inicia nuevos cambios en el territorio. Sobre estas bases, analiza los procesos de cambio en las percepciones y relaciones con el medio ambiente y la tierra en la comunidad campesina afectada por la extracción de hidrocarburos.

Cierra este texto Mauricio Hernández Pérez quien en el Epílogo imprime su característico sello crítico y reflexivo en una invitación a continuar repensando estos temas.

En suma, se espera que el lector disfrute del potencial heurístico de una obra que encuentra su valor agregado no sólo en la calidad académica y experticia de sus autores sino por sobre todo, en su rasgo más resaltante: un amplio eclecticismo que combina saberes, metodologías y lenguajes que permiten arrojar miradas múltiples sobre un fenómeno inabordable exhaustivamente por su complejidad.

María Eugenia Vega

Hugo Fernando Guerrero Sierra Pedro Mauricio Acosta Castellanos Bogotá, octubre de 2018 
